Lexis Vol. XLV (2) 2021: 865-890

\title{
Incaísmo, indigenismo y gamonalismo: Rumi Maki desde tres modalidades escriturales de representación
}

\author{
César Coca Vargas \\ 0000-0002-9548-1497 \\ The Graduate Center - City University of New York \\ ccoca@gradcenter.cuny.edu
}

\section{RESUMEN}

El 2 de diciembre de 1915, en Azángaro (Puno), se produjo un levantamiento campesino en la hacienda San José. Las fuentes que registraron el acontecimiento indican que la sublevación tuvo la presencia de un líder de inspiración incaica autodenominado "Rumi Maki Ccori Zoncco". De esta figura legendaria, cuyo nombre real habría sido Teodomiro Gutiérrez Cuevas (1864), se ocuparon desde escritores y periodistas contemporáneos al suceso hasta investigadores posteriores. Aunque se pueda observar, en algunos casos, un análisis de enfoque cultural (incaístas o indigenistas, por ejemplo), considero que no ha existido una explicación detallada de las implicancias culturales de aquellos quienes dedicaron líneas a la figura de Rumi Maki. Por esta razón, el propósito principal de mis reflexiones lee el levantamiento de San José en esa dirección. Para esta finalidad, utilizaré tres modalidades escriturales: la incaísta (José Carlos Mariátegui), la indigenista (Dora Mayer, Manuel González Prada, Francisco Chukiwanka) y la gamonalista (Sebastián Urquiaga, Angelino Lizares). A partir de aquí, me detendré en analizar el tipo retórico de cada modalidad escritural que representó a Rumi Maki.

Palabras clave: Rumi Maki, incaísmo, indigenismo, gamonalismo

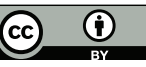

https://doi.org/10.18800/lexis.202102.011 
Incaísmo, indigenismo y gamonalismo: Rumi Maki from Three Rethorical Types of Representation

\begin{abstract}
On December 2, 1915, in Azángaro (Puno), there was a peasant uprising on the San José farm. The sources that recorded this event indicate that an Inca-inspired leader who called himself "Rumi Maki Ccori Zoncco" participated in said rebellion. Contemporary writers and journalists and even subsequent researchers have written about this legendary figure, whose real name might have been Teodomiro Gutiérrez Cuevas (1864- $\dot{\imath}$ ?). Although in some cases we can observe analyses from a cultural approach (incaistas or indigenistas, for example), I believe that there has been no detailed explanation of the cultural implications from those who dedicated lines to Rumi Maki's figure. For this reason, my main purpose is to read the San José uprising in that direction. To this end, I will use three rhetoric modalities: the incaista (José Carlos Mariátegui), the indigenista (Dora Mayer, Manuel González Prada, Francisco Chukiwanka) and the gamonalista (Sebastián Urquiaga, Angelino Lizares). From here on, I will analyze the the rethorical type of each writing modality that represented Rumi Maki.
\end{abstract}

Keywords: Rumi Maki, incaismo, indigenismo, gamonalismo

\title{
O. Palabras iniciales
}

El 20 de mayo de 1916, la revista semanal Variedades, dirigida por Clemente Palma, dio a conocer dos ilustraciones que tenían como protagonista a Rumi Maki. En realidad, eran dos caricaturas de la clásica sección de la revista llamada "La semana cómica”. En esas dos caricaturas se observa un hombre de aproximadamente cincuenta años, vestido con poncho, sandalias y una especie de mask'aypacha ${ }^{1}$ sobre la que se levantan dos plumas; en el fondo, detrás de una ventana, se encuentra la imagen del sol. Rumi Maki, el protagonista,

\footnotetext{
1 Durante el Tawantinsuyu, la "mask'aypacha” fue un conjunto de trenzas con incrustaciones de oro y plumas que simbolizó el poder del inca supremo (el sapa inca).
} 
yace en una prisión con el rostro desencajado. Las dos leyendas de dichas ilustraciones recrean cortos diálogos entre él y el sol.

En la primera ilustración, denominada "Rumimaqui en su prisión", el protagonista le suplica al sol que no lo abandone "a la ferocidad de los civilizados". La respuesta que recibe es una negativa: el sol -el "padre mío" de Rumi- reconoce que ya no posee la capacidad para salvarlo, que ya no es más "el todopoderoso del imperio de los Incas". En la segunda caricatura, nombrada "Rumimaqui y la justicia”, el diálogo continúa: “¿Oye, Padre? El soberano de la ciudad de Pizarro me acusa del enorme delito de traición a su patria”. El sol replica, sin dejar de reconocer irónicamente su pena, que debería existir enjuiciamiento y encarcelación para todos los falsos reformadores y los falsos restauradores de la libertad.

He deseado iniciar con las reflexiones anteriores no con la idea de definir el perfil ideológico o las aspiraciones culturales de Variedades, pues escapa a mis propósitos en este trabajo. Sin embargo, a partir de allí quiero destacar el modo en que la revista abordó la figura de Rumi Maki, el cual a su vez permite identificar características formales de las representaciones discursivas que se hicieron sobre ese referente. De esta manera, la excusa de las caricaturas revela dos particularidades: en primer lugar, su distancia tanto geográfica como identitaria del referente real "Rumi Maki"; en segundo lugar, su connotación semántica de extrañeza: una lectoría de usos culturales elitistas frente a un significante "incaico". La característica determinante de esas caricaturas es su tono sarcástico a partir del cual se puede afirmar que sus significaciones no están restringidas al ámbito ilustrativo, sino que, en cambio, pueden ser interpretadas desde el horizonte ideológico.

En una cárcel arequipeña, en el año 1916, un hombre (de edad similar al hombre de las caricaturas) estuvo preso luego de presuntamente haber liderado una sublevación campesina en Puno, acusado de traidor a la patria. El nombre de ese supuesto restaurador fue Teodomiro Gutiérrez Cuevas (1864-¿ ¿ ?), quien, según la crítica que le ha dedicado páginas (Augusto Ramos Zambrano 2016, Mauro Paredes 1970, José Tamayo Herrera 1982), asumió la denomina- 
ción quechua Rumi Maki Ccori Zoncco, cuyo significado podría ser traducido como "Mano de piedra, corazón de oro". A pesar de la importancia del hecho real², es decir, de la sublevación campesina ( sin importar la magnitud) de San José en Puno, en mis indagaciones quiero destacar otro "hecho". Me refiero a la representación escritural que se realizó de Teodomiro Gutiérrez / Rumi Maki durante aquellos años. No mencionaré todas las que hubo, por supuesto, sino que resaltaré tres modalidades escriturales (la incaísta, la indigenista y la gamonalista) para intentar comprender el fenómeno "Rumi Maki”.

\section{CCORI ZONCCO}

En los años finales del siglo XIX y los primeros años del siglo $\mathrm{XX}$, hubo una expansión económica en algunas regiones del sur peruano a través de las tecnologías de la modernidad (en particular, el ferrocarril). De esta manera, Puno ingresó en el radio comercial del circuito sureño que, por ese entonces, tenía como centro económico a la ciudad de Arequipa. José Tamayo Herrera, quien estudió la historia social de Puno con detalle, aseguraba que la introducción del ferrocarril en 1875 le dio a la región la posibilidad del comercio lanero. Sin embargo, continuaba Tamayo, el impacto del ferrocarril se vio interrumpido por la guerra que sostuvo el país contra Chile (1879-1883) (1982: 87). Aunque las aristas de desarrollo económico pueden ser múltiples y muy complejas de explicar, el mismo investigador afirmaba que durante esos años se produjo el renacimiento cultural más interesante de la historia del Puno republicano.

Estoy parcialmente de acuerdo con lo mencionado por Tamayo, pero considero que habría que ir con algunas precauciones nece-

2 El significado que asigno a "real" no se encuentra supeditado a ningún horizonte epistemológico ni mucho menos. Lo empleo, en cambio, simplemente como un sinónimo de verídico o verdadero. En este sentido, cuando hablo del Teodomiro Gutiérrez "real" me refiero expresamente a aquel hombre de carne y hueso que pudo haber liderado una sublevación en el sur peruano.

3 Titulo a este apartado "Ccori Zoncco" con el fin de sintetizar alegóricamente la personalidad de Teodomiro Gutiérrez Cuevas en su etapa como subprefecto. 
sarias, sobre todo si se desea entender a cabalidad el impacto de ese "renacimiento cultural puneño". En primera instancia, habría que preguntarse qué tipo de irradiación cultural existió previamente para sostener que hubo un renacimiento en el siglo XX. En segunda instancia, si se asume como verdadero el renacimiento, habría que interpretar con mesura su naturaleza, pues sería necesario saber si tuvo implicancias en un horizonte social amplio o más bien fue un fenómeno extremadamente aislado. Me inclino a pensar en lo segundo, ya que no se puede hablar de estructuras culturales en el Puno de entonces. En otras palabras, considero que Tamayo exageraba cuando habló de un renacimiento puneño.

Me imagino que el fenómeno en el que estaba pensando el mencionado autor era el grupo Orkopata (ca. 1926) y su conocido boletín ${ }^{4}$. Gamaliel Churata, líder del grupo, fue una aparición singular en la historia de Puno, debido a que sacó provecho de algunas condiciones particulares de la época. Precisamente una de ellas fue el poco dinamismo cultural del altiplano, el cual le brindó posibilidades inimaginables de impacto, aunque el mismo estuvo restringido a la intelectualidad. Como se ha podido notar, el año de aparición del mencionado grupo es tardío respecto de la cronología del propio Tamayo, pero es la muestra más llamativa de ese denominado "renacimiento cultural". En Puno, retomando el hilo anterior, no existieron estructuras culturales (ni pedagógicas ni educativas), sino apariciones esporádicas y de relativa influencia. En este sentido, ya entrado el siglo XX, José Antonio Encinas fue, al igual que Arturo Peralta (Gamaliel Churata), otro de los intelectuales más notables de esa ciudad.

José Deustua y José Luis Rénique manifestaron que la expansión económica y la renovación intelectual no transformaron constitutivamente la base rural del Perú. Por el contrario, consideraron que en las poblaciones andinas se mantenían constancias y permanencias culturales milenarias, al menos en algunas regiones (1984: 16-17).

4 Me estoy refiriendo al Boletín Titikaka (1926-1930), el cual ha sido estudiado en abundancia. 
Finalmente, Deustua y Rénique creyeron que, en las primeras tres décadas del siglo XX, en el país primaba el inmovilismo sobre la movilidad. A pesar de que ellos matizaron ciertamente la idea en su libro dedicado al indigenismo en el periodo 1897-1931, creo que el término “inmovilismo" no fue el más afortunado. La inauguración del ferrocarril en el sur peruano tuvo repercusiones inmediatas en las esferas sociales y, aunque en menor medida, también en las culturales de Puno. Una prueba de ello es el caso de Teodomiro Gutiérrez Cuevas, quien en los primeros años del siglo XX ocupaba el cargo de subprefecto de Chucuito, en la ciudad de Puno.

Las referencias biográficas sobre Teodomiro Gutiérrez coinciden en que estuvo a cargo de la subprefectura de la provincia puneña de Chucuito entre los años 1903 y 1904 (Arroyo Reyes 2005: 127; Zambrano 2016: 349). Aseguran, asimismo, que Gutiérrez tuvo una actitud favorable hacia el campesinado ${ }^{5}$ : desde la defensa que asumió por reconocerles su dignidad como personas hasta las propuestas que realizó en torno a la educación de la población de las serranías. El trazo biográfico de Gutiérrez permite que se identifique un primer momento de su contacto con la problemática campesina; en otras palabras, faculta que se pueda entender su posterior participación en el levantamiento de San José del año 1915․ De esta manera, el arco temporal de su vida partiría de su condición de reformador (a través de los tres cargos más importantes que cumplió ${ }^{7}$ y concluiría en su etapa de compromiso: la de

\footnotetext{
5 De ahora en adelante, salvo contadísimas excepciones, emplearé el término "campesinado" para referirme indistintamente a "realidad andina" o "población andina”. A pesar del sesgo marxista, en clave mariateguiana, que el término ha recibido, mi uso estará restringido a las equivalencias anteriores.

${ }_{6}$ Quisiera aclarar que seguiré las representaciones discursivas que se hicieron sobre el protagonista. Esto es, 1) que consideraré a Gutiérrez Cuevas y a Rumi Maki como dos instancias complementarias y problemáticas; 2) que diré que hubo un levantamiento campesino con participación de alguien llamado Rumi Maki; y 3) en conclusión, que existió “alguien” sobre el que se sostuvo la idea del Rumi Maki restaurador del Tawantinsuyu (idea, insisto, que puede ser verdadera o no).

7 Teodomiro Gutiérrez Cuevas fue dos veces subprefecto: en Chucuito y en Huancayo. Además, fue comisionado en Samán por encargo del presidente peruano Guillermo Billinghurst (1913).
} 
restaurador simbólico (por medio de las referencias incaístas que rodearon la existencia de Rumi Maki).

Los levantamientos campesinos de esos años fueron calificados por Alberto Flores Galindo (1987) y Tamayo Herrera (1982) como la "gran sublevación del sur" y la "explosión de la indiada”, respectivamente. La historiografía que se ha ocupado de las luchas campesinas del sur peruano (en particular, Puno), no cuantiosa pero sí importante, coincide en que la posesión de tierras fue el punto crítico y el movilizador social más relevante entre la población andina de aquellos años. Al igual que Churata o que Encinas, Teodomiro Gutiérrez fue una singularidad en Puno, mucho más aún si se destaca su participación proandina dentro del aparato estatal de las provincias peruanas de entonces.

Carlos Arroyo Reyes se ocupó del primer Gutiérrez en una sección de su libro sobre la década de 1910, la cual tituló "Un militar indigenista”. Allí destacó la posición “proindígena” del entonces subprefecto de Chucuito y afirmó, además, que “decretó la abolición de los servicios forzosos y gratuitos que se le imponían prepotentemente a los indios" (Arroyo Reyes 2005: 127). A partir de aquí, parece cierto que Gutiérrez asumió su tarea con dedicación y con exigencias de justicia. No solo criticó a los terratenientes y a los gamonales ${ }^{8}$, sino también algunos hábitos de la población serrana. Carlos Contreras y Jorge Bracamonte (1988) tuvieron acceso a documentos inéditos, escritos de la propia mano de Gutiérrez cuando ocupó el cargo de subprefecto de Huancayo, en la sierra central del Perú. Su llegada a esa región peruana se dio inmediatamente después de su destitución de Chucuito, ocurrida en agosto de 1904. Antes de ingresar al detalle de esos documentos, creo que es necesario conocer las repercusiones de su paso por Chucuito.

En primer lugar, deseo destacar que su presencia en dicha provincia causó rechazo entre los sectores terratenientes y eclesiásticos, ya que la destitución que sufrió se dio por la intervención de

\footnotetext{
8 José Tamayo Herrera trató con detalle las diferencias entre los términos "terrateniente” y “gamonal” (1982: 149-170).
} 
los gamonales en el parlamento peruano. En otras palabras, la representación política que ellos poseían cumplió el pedido de relegarlo de su cargo. Esta clase de acciones, al contrario de haber simbolizado una preocupación nacional de gran escala, fue un ejemplo evidente de constantes y sistemáticos actos de abuso de poder de las oligarquías provincianas. A partir de aquí también puede entenderse el inestable apoyo institucional que recibió de los dos presidentes que le otorgaron confianza, Manuel Candamo (19031904) y Guillermo Billinghurst (1912-1914). En resumen, su labor de subprefecto representó un peligro para un sector terrateniente con importante presencia institucional.

En segundo lugar, quiero resaltar la semblanza de Teodomiro Gutiérrez elaborada por Manuel González Prada, probablemente en el año 1905. Ese texto, titulado “Autoridad humana”, apareció en la revista limeña El Indio de manera anónima. Hay varios aspectos de ese breve artículo que llaman la atención (su naturaleza anónima, por ejemplo), pero considero pertinente iniciar con una reflexión acerca del origen del texto. Es importante mencionar la fecha prematura en que fue compuesto: por los mismos meses en que Gutiérrez desempeñaba su labor. En otras palabras, es de suma relevancia subrayar que su tarea de subprefecto, a pesar de la lejanía geográfica respecto de Lima, no pasó desapercibida por los escritores capitalinos; más adelante, incluso Dora Mayer y José Carlos Mariátegui escribieron sobre su participación en la sublevación de San José. Sin embargo, la semblanza de González Prada es notoria debido a la contemporaneidad con un Gutiérrez Cuevas sobre el que aún no se había creado la imagen apostólica o incásica.

“Autoridad humana" inicia resaltando la excepcionalidad de Gutiérrez a partir de una retórica de complementariedad antagónica, es decir, recreando el contexto social de los pueblos serranos con el propósito de resaltar al entonces subprefecto. Es más, González Prada utilizó en ese breve texto el implicativo de la primera persona en plural para reconocer lo que podría entenderse como una problemática nacional: "Vivimos acostumbrados a que los pueblos se duelan de sus malas autoridades y eleven inútiles 
reclamaciones al Gobierno" (1986: 299). Dicha retórica antagónica tiene dos marcas de importancia que pueden ser detectadas con el título de la semblanza y con el cierre del texto que, al mismo tiempo, alegorizan dos momentos de la vida de Gutiérrez.

En el título de su texto, González Prada utilizó los significantes “autoridad” y "humana", los cuales formaron parte del discurso paternalista de las prácticas indigenistas de fines del siglo XIX e inicios del siglo XX. En otros términos, la inclusión de la población andina, bajo el título "Autoridad humana”, debía darse por el reconocimiento de una presencia jerárquicamente superior que tuviera la sensibilidad —el "afecto indigenista" - por abolir las injusticias y los maltratos que recibía el campesinado. En palabras del autor de la semblanza, Gutiérrez Cuevas representaba esa autoridad que impartía leyes humanas que desarraigaban costumbres feudales (1986: 300). La singularidad de Gutiérrez radicaba en su carácter de precursor, ya que su trabajo oficial rompía con la regla generalizada de abuso.

El cierre de la semblanza es mucho más interesante. Allí González Prada pareció anunciar un levantamiento de proporciones restauradoras y milenarias. La asociación entre gobierno y raza que González Prada realizó ingresa en los códigos indigenistas posteriores, como los de Luis E. Valcárcel. Aunque en él no exista la radicalidad mesiánica de Valcárcel, el presagio de González Prada es contundente y sería materializado, una década más tarde, en la figura apostólica de Rumi Maki: "Existe muchísima diferencia entre el motín de una soldadesca y el levantamiento de una raza para sacudir el yugo y vengar las iniquidades de tres o cuatro siglos” (1986: 301). De esta manera, lo que inicia con el despliegue de una retórica más bien paternalista (la singularidad de la protección de la "raza indígena”) concluye con una profecía de índole incaísta (el advenimiento de la raza).

La mirada de González Prada puede ser calificada como una lectura referencial, es decir, como la interpretación discursiva de un hecho real. Teodomiro Gutiérrez Cuevas era esa marca referencial sobre la que se trazó una imagen verosímil de impacto político y 
social. En el texto de González Prada se aprecia una lectura de la imagen de Gutiérrez Cuevas en sus instantes incoativos y en los momentos potenciales de una futura restauración tawantisuyense. A pesar de que su labor en Chucuito se vio interrumpida por los diputados puneños en Lima, volvió a ser nombrado subprefecto en octubre de 1906, esta vez de Huancayo.

Esa etapa “institucional” está determinada con notoriedad en un documento - llamado por sus descubridores "memoria" - del subprefecto de Huancayo, Teodomiro Gutiérrez. Carlos Arroyo Reyes aseguró que su trabajo estuvo caracterizado por la movilidad y el conocimiento de primera mano al que accedió por medio de sus viajes y conversaciones con habitantes de las provincias serranas (2005: 130). Esas primeras ligazones con dirigentes campesinos sirvieron de base social para su tentativa posterior. Además, las consecuencias de ese aprendizaje fueron reconocibles en un informe de matices etnográficos que el propio Gutiérrez realizó y que, en palabras de Contreras y Bracamonte, buscó “reflexionar sobre las causas de 'las condiciones desgraciadas' en que se desenvolvía la vida indígena” (1988: 7). Sin embargo —de acuerdo con esos mismos investigadores - también tuvo la voluntad de proponer soluciones para mejorar esa condición de desgracia. La razón por la que el informe es especial se debe a que allí se encuentran pasajes en que Gutiérrez emitió juicios de valor negativos respecto de ciertas costumbres de la población andina.

Dichas opiniones problematizan más aún la imagen de Gutiérrez, ya que no se puede afirmar, con plena seguridad, que su característica distintiva haya sido la férrea defensa de las sociedades andinas. Por el contrario, al leer el informe se observa que existió cierto respaldo al modelo social que prevalecía por aquellos años. Para Gutiérrez Cuevas, el sistema no era completamente inadecuado; más bien culpaba a los elementos que lo conformaban: las autoridades abusivas. De este modo, su informe fue un texto de crítica doble, caracterizado por el anhelo de que el país pueda ser uno moderno, de leyes que se respeten y de vicios que se eliminen. Contreras y Bracamonte resumieron la postura de Gutiérrez en 
Huancayo como un rechazo de dos direcciones: a "los abusos de los gamonales, como también [a] la situación de inmoralidad e ignorancia en que viven los indios" (1988: 9).

El dispositivo retórico preferido por Gutiérrez Cuevas en su informe fue la personificación del "mal". Así pues, su texto provee algunas imágenes de secuencia significativa en esa dirección: gobernadores - criminales - violencia - abuso. A partir de aquí, creó un discurso que tuvo como punto definitivo esa personificación. Su crítica no emitió más alegorías que esa, pues, a pesar de las variaciones que realizó sobre los protocolos de los informes burocráticos, apuntó más bien a una reforma de orden administrativo (en este sentido, conservador). Por esta razón, lo que se observa se distinguió por haber sido una recreación informativa. A estas alturas, quisiera entender el informe desde la noción de "simpatía indigenista”, amparada en la figura del subprefecto Gutiérrez. Teodomiro Gutiérrez marcó cierta distancia de la causa que defendió a través del gesto paternalista, es decir, por medio de una defensa jerarquizada de la población andina. De este modo, la posición ambivalente del entonces subprefecto puede explicar el carácter progresista de un sector del indigenismo.

Lo que deseo destacar de esto último es un principio básico del indigenismo artístico; esto es, la representación de "lo otro". Gutiérrez Cuevas se sabía superior, tanto en el orden institucional como en el social, y por ese motivo se embargó en la tarea de rechazar aspectos que, a sus ojos, eran premodernos. Ese principio fue uno de los móviles más activos de la discusión en torno al llamado "problema del indio" desde inicios del siglo XX peruano. Sin embargo, debo reconocer que Gutiérrez no pretendió, en su informe al menos, elevar su texto a la categoría de "artístico". Por el contrario, su gesto paternalista tuvo una pretensión de naturaleza social y judicial. La defensa del campesinado debe entenderse desde la perspectiva del subprefecto Gutiérrez- como un destierro de los "vicios y el estado anormal" (Contreras y Bracamonte 1988: 18) en que se encontraba — según su posición — la población andina. 
Carlos Arroyo Reyes realizó el balance de la primera etapa de Teodomiro Gutiérrezy aseguró que sus ideas no guardaban relación con la revolución agraria, sino más bien con la reforma de un conjunto de medidas administrativas y legislativas que el Estado debía proveer (2005: 136-137). De este momento inicial, me ha interesado subrayar la importancia de Gutiérrez en tanto representante del aparato estatal. Además, me ha parecido relevante mencionar el hecho de que el referente de Gutiérrez es inteligible, a pesar de que no abunde la información de su labor como subprefecto de dos regiones del Perú.

La excepcionalidad de este primer Gutiérrez se debe a que no reprodujo los códigos de socialización dominantes (abuso, intimidación, maltrato, etc.) en los distritos de presencia terrateniente. Su trabajo de subprefecto, sin embargo, no fue la única participación “indigenista” que tuvo por aquellos años: a finales de la década de 1900, participó de los conversatorios de la Universidad de San Marcos y, desde 1910, integró la directiva de la Asociación Pro Indígena de Lima. Precisamente —cuando la imagen de Gutiérrez Cuevas se fue diluyendo para dar paso a la leyenda de Rumi Makiel boletín mensual de la Pro Indígena dedicó editoriales, informes y artículos de opinión a los hechos de Azángaro. Sus lazos con la directora del boletín, Dora Mayer, y el delegado de la asociación en Puno, Francisco Chukiwanka, fueron momentos de intervención directa y activa en la "causa indígena”.

Antes de iniciar mis reflexiones sobre Rumi Maki, considero pertinente realizar una breve síntesis. En primer lugar, la intervención de Gutiérrez Cuevas en las dos subprefecturas fue clave para su toma de conciencia. En segundo lugar, las conversaciones que tuvo con las poblaciones andinas le permitieron acceder a imaginarios particulares del ande. En tercer lugar, sus viajes le brindaron el conocimiento para rechazar los "defectos" de esos mismos imaginarios. Por último, la posición indigenista de Gutiérrez Cuevas no fue ortodoxa, sino más bien de una plasticidad selectiva: para él, existían prácticas andinas negativas que debían ser desaparecidas si a lo que se aspiraba era al progreso. De esta manera, me gustaría 
concluir este apartado con Contreras y Bracamonte, quienes sostuvieron que hubo un cambio en el pensamiento de Gutiérrez a partir de su seminal presencia en las subprefecturas: "[P]robablemente su contacto posterior, más prolongado, con la realidad del sur andino, tan distinta en varios aspectos con la sierra central, haya producido cambios importantes en el pensamiento de una persona tan sensible como él” (1988: 11).

\section{LA MASK’AYPACHA9}

El 25 de abril de 1917, en el periódico limeño El Tiempo, José Carlos Mariátegui publicó una breve nota titulada "Minuto solemne". La misma estaba dedicada a lo que para Mariátegui significaba el "renacimiento peruano", es decir, el advenimiento de un tiempo fundacional, cuyo cimiento había que encontrarlo en el incario. En su opinión —aunque algo hiperbólica-, en el Perú de inicios del siglo XX, el arte, la música y el teatro de inspiración incaísta materializaban dicho renacimiento. No obstante, Mariátegui aseguraba - por si fuera poco- que los incas habían salido de las huacas para combatir a la oligarquía limeña representada en la dinastía Pardo ${ }^{10}$. Esos incas — continuaba el Amauta- eran dirigidos por el sumo sacerdote del sol, el general Rumimaqui, que deseaba ser el inca portador de la mask'aypacha

La nota de Mariátegui puede ser caracterizada de diversas maneras, pues transita desde el tono dramático hasta el acento jocoso. Sin embargo, para los objetivos de mis reflexiones resulta conveniente señalar que su texto despliega toda una retórica incaísta, a partir del uso de una terminología de cosmovisión andina

\footnotetext{
De modo similar al título del apartado anterior, en este empleo el nominal “mask'aypacha” para subrayar las representaciones que se hicieron sobre Rumi Maki como un restaurador incaico.

10 La familia Pardo poseía en el Perú una antigua data colonial, la cual a su vez le permitió ocupar cargos de poder e influencia política. Hacia finales de 1871, Manuel Pardo fundó el Partido Civil y al año siguiente fue elegido presidente, el primero de orden constitucional en el país. El civilismo comandó la política nacional desde entonces por casi cinco décadas.
} 
(huacas, mask'aypacha, sol, etc.). La onda del sur, como la llamó Mariátegui ${ }^{11}$, o la explosión de la indiada, en términos de Tamayo Herrera, se consolidó por aquellos años como parte del discurso tawantisuyense de la restauración que llevaba a cabo Rumi Maki. La síntesis de la codificación de todos estos referentes fue empleada por varios tipos escriturales, de los cuales deseo destacar tres: el indigenista, el incaísta y el gamonalista.

Hablo de tipos escriturales en el sentido de que el referente Rumi Maki fue objetivado de tres modos diversos, o de tres estilos de representación escrita. Históricamente, los tipos indigenista, incaísta y gamonalista se superpusieron y establecieron, incluso, un enfrentamiento entre ellos. De lo que se trató en realidad fue de situar a Rumi Maki en el imaginario político y social de los sectores que se encontraban involucrados en el problema “indígena”. En ese sentido, el registro indigenista y el registro gamonalista fueron posiciones enfrentadas a partir de su tratamiento de la población andina. El primero fue paternalista e integrador: buscaba, pues, la inserción del mundo andino al proyecto nacional criollo y muchas veces estuvo amparado en el cuidado y la compasión por el explotado. El segundo, por el contrario, pretendía mantener el status quo de la situación oprimida de la población de los Andes; en esta dirección, se hallaba gestionada y alentada por quienes poseían intereses económicos. El tipo incaísta fue un registro particular, asociado más bien a las esferas intelectuales limeñas que veían como un "motivo de inspiración literaria” la existencia de un restaurador inca. Debido a esto, su escritura se caracterizó por sus claras connotaciones y alegorías al Tawantinsuyu.

Durante la primera etapa de su vida institucional, Teodomiro Gutiérrez Cuevas fue un referente identificable, ya que integraba el aparato estatal. A pesar de las contiendas que sostuvo con varias autoridades provincianas, cuya representación parlamentaria obstruyó su trabajo, su presencia nunca dejó de estar en el radio

\footnotetext{
11 Aquí aludo a otra nota, llamada “Grimas y zozobras”, que publicó en El Tiempo el 17 de enero de 1917.
} 
de "lo real". El nombramiento que recibió por parte de Guillermo Billinghurst parece ser el instante preciso en que su imagen dejó de ser netamente referencial para convertirse en una figura escurridiza. Gutiérrez llegó a Puno en setiembre de 1913 para investigar los asesinatos de Samán y, a raíz de esta nueva experiencia, elaboró un informe detallado de lo acontecido alli1 ${ }^{12}$. Es más, Gutiérrez acompañó a una delegación puneña que se entrevistó con el propio Billinghurst a finales de 1913 en Lima. El registro de esa visita, consolidado en una histórica fotografía, estuvo a cargo del diario La Crónica ${ }^{13}$.

En el apartado anterior resalté la relevancia de sus años como subprefecto. Principalmente porque le brindó la posibilidad de acceder a la realidad campesina sin la mediación periodística, aunque claro está que él jamás dominó el quechua. Gracias a esa experiencia, se puede comprender su cercanía con los líderes campesinos de la delegación puneña y, más adelante, sus estrechos vínculos no solo con los letrados Francisco Chukiwanka y Julián Palacios, sino también con José María Turpo, dirigente campesino de influencia notoria entre la población de Azángaro. Turpo participó en el levantamiento de San José, luego del cual fue asesinado en condiciones inhumanas. Este levantamiento (se desconoce con precisión su magnitud) ocurrió entre el 1 y 2 de diciembre de $1915^{14}$, es decir, cuando ya Gutiérrez Cuevas había escapado de la refe-

\footnotetext{
12 Se ignora el contenido de ese documento. Gutiérrez Cuevas declaró incluso que fue enviado al extranjero para su publicación en una prestigiosa revista norteamericana. Hay que recordar que la conclusión de su informe coincidió con el golpe de estado que recibió Billinghurst. Probablemente, entre los documentos saqueados o robados, se encontraba el suyo.

13 La imagen corresponde a la portada del 30 de noviembre de 1913, en la que apareció el niño puneño Cirilo Calloaspaza.

14 Desde finales de noviembre de 1915 se venía escribiendo sobre las agitaciones en Puno. Algunos periódicos de Arequipa y de Puno registraron el hecho. Por ejemplo, el 26 de noviembre El Eco de Puno publicó un artículo titulado "Los indios en movimiento". Incluso algunos periódicos ya hablaban de un tal Rumi Maki, antes de que aconteciera el asalto a San José.
} 
rencialidad concreta. Desde esos instantes, no existe información plenamente certera de sus actividades ${ }^{15}$.

He denominado modalidades escriturales a aquellos textos que se caracterizaron por haber dado una versión de Rumi Maki bajo una retórica particular. Por ejemplo, "Minuto solemne” y "Grimas y zozobras" de Mariátegui son modalidades escriturales de una propuesta discursiva incaísta. En otras palabras, Mariátegui, en esos textos, rechazó cualquier compromiso fáctico con la "cuestión indígena” y, por el contrario, reprodujo los códigos modernistas (en su tono exótico) que abordaban por aquel entonces el mundo andino $^{16}$. Sin embargo, las notas de Mariátegui, que corresponden a 1917, fueron más bien el colofón de una serie de tipos escriturales que se dieron desde que Gutiérrez Cuevas asumió la subprefectura de Chucuito. Rumi Maki (o lo que es mejor, su significante), no obstante, se dio a conocer en 1915, aunque pueda rastrearse su gestación un par de años antes. Augusto Ramos Zambrano remarcó que, en los días finales de setiembre del año 1913, Gutiérrez Cuevas, quien se dirigía a Samán por encargo de Billinghurst, distribuyó un volante escrito íntegramente en quechua en el que se reconocía un comisionado especial nombrado por el entonces presidente peruano, con el fin de iniciar las averiguaciones: "Tuqyuta yachaciyta munani allinin qupajj mana maykenman sayakusps tuquyniyqichista imanqajjta uyariskayqichis"17 (Ramos 2016: 350).

No pretendo ingresar en asuntos de índole histórica ni mucho menos a la verificación exhaustiva de cuándo surge el imaginario Rumi Maki. Sin embargo, sí deseo destacar el impacto que pudieron

15 No he podido comprobar la veracidad de un hallazgo de Augusto Ramos Zambrano (2016: 405-415). Se trata de una carta de Potosí escrita en 1937, supuestamente por alguien que se hizo llamar general Huayna Cápac. En ella se adjuntaba un plan de dieciocho puntos de restauración del Tawantinsuyu. Ramos Zambrano, siguiendo lo asegurado por ese general, sostuvo que el autor del plan fue Rumi Maki. Debido a que tengo algunas sospechas de esta información, no haré ninguna mención a ella en este trabajo.

16 Abraham Valdelomar fue quizás el más notorio representante de esta modalidad en la literatura peruana. Sus relatos ambientados en el incanato, reunidos póstumamente en Los hijos del sol, son una muestra de ello.

17 "Quiero que sepan que mi deseo es escucharlos; imparcialmente, a todos" (traducción del propio Ramos Zambrano). 
haber cumplido gestos como el de comunicarse en quechua con la población campesina. Así pues, Julián Palacios cumplió una tarea sumamente importante, pues fue él quien trabajó de intermediario entre Gutiérrez Cuevas y los líderes campesinos. Por esta razón, la modalidad escritural que representó Mariátegui en sus dos breves notas no fue la única que tuvo repercusión inmediata. Las relaciones de Gutiérrez con los habitantes de Puno tuvieron dos consecuencias naturales: 1) su participación como miembro/delegado de la Pro Indígena; y 2) su enemistad con el sector gamonal. En estos dos casos existió un intento de representación discursiva sobre lo ocurrido en San José; en otras palabras, los voceros periodísticos del gamonalismo y del indigenismo cumplieron propósitos de acuerdo con sus propios intereses.

De esta manera, el periódico Elindio, propiedad del terrateniente/ gamona $1^{18}$ Angelino Lizares Quiñones en Puno; y el mensuario El deber pro indígena, dirigido por Dora Mayer en Lima, se ocuparon, respectivamente, de exaltar la idea de una sublevación milenaria del Tawantinsuyu y de negar la existencia mesiánica de Rumi Maki. Estas dos posturas respecto de un mismo acontecimiento representan, al mismo tiempo, dos tipos escriturales diferentes, lo cuales podrían situarse - para el caso del primero- en el horizonte gamonalista; y - para el caso del segundo- en la línea indigenista. No obstante, debo admitir que no fueron las únicas manifestaciones verbales de la posición que cada grupo defendía, sino que existían también instancias gubernamentales sobre las que se disputaba la "cuestión indígena" (a la cual estuvo vinculada la representación Rumi Maki). Por ejemplo, los diputados puneños se desenvolvieron en el espacio parlamentario, mientras que (aunque con mucho menos fuerza que los anteriores) los "pro indígenas" desarrollaron su labor en las esferas judiciales.

18 Coloco esta distinción entre uno y otro término debido a que no siempre actuaron como sinónimos directos. Para una explicación pormenorizada, como ya recomendé, ver el trabajo de Tamayo Herrera (1982), titulado Historia social e indigenismo en el altiplano, en particular, la sección "La etiología del gamonalismo y la mentalidad gamonal" (150-190). 
En enero de 1916, el vocero de la Pro Indígena dedicó un editorial en el que se realizaba un balance de los sucesos de San José. Según ese texto, luego de haber contrastado información ${ }^{19}$, Mayer llegó a la conclusión de que no se había dado sublevación alguna, sino que había una conspiración de gamonales. Para El Deber Pro Indígena, el objetivo era "reducir a la indiada a la condición esclava" (193), a partir de un sesgo de determinismo biológico: los "indígenas" eran inferiores. Las líneas finales de ese editorial pueden ser leídas en sintonía con los artículos de opinión que publicó Dora Mayer en El Comercio en enero de ese mismo año ${ }^{20}$. El primero de ellos, "La trama de la sublevación indígena" presenta características sobresalientes en cuanto a la perspectiva que Mayer adoptó sobre Rumi Maki. En ese texto, la modalidad escritural de su autora puede ser calificada de indigenista (muy lejos del exotismo incaísta de Mariátegui). Para esta finalidad, rechazó toda existencia de un caudillo externo llamado Teodomiro Gutiérrez o Rumi Maki. Su retórica tenía como pretensión última la desestimación de la leyenda mesiánica.

Dora Mayer contrapuso dos significantes en sus dos notas: trama (conspiración gamonal) y masacre (genocidio gamonal). El segundo artículo, "La masacre de San José", permite entender las implicancias de su lógica discursiva a partir del contrapunto de esos dos significantes. En ambos casos, el referente de inspiración es Gutiérrez / Rumi Maki, debido a que el tipo escritural del gamonalismo había conseguido erigir el imaginario de que efectivamente existía una sublevación tawantisuyense. La contienda de Mayer, entonces, tenía que situarse en el orden referencial del hecho, relegando la idea de una fantasía incaica. Resulta llamativo el procedimiento escritural de la directora de El deber pro indígena en sus dos textos, pues pretendió desbaratar la materialidad de Gutiérrez / Rumi Maki

19 El mecanismo del contraste fue empleado en reiteradas oportunidades por los integrantes de la Asociación Pro Indígena, ya que, al poseer delegados en diversas regiones del país, podían comparar su propia información con la oficial y/o la hegemónica.

20 Hay que decir, sin embargo, que la composición de esos artículos se dio en diciembre, solo unos días después del levantamiento de San José. 
para dar paso a la abstracción de unos valores que - en palabras de González Prada- estarían ejemplificados en el entonces subprefecto. En "La masacre de San José”, Mayer dictaminó:

El indio no tiene abogados, no tiene caudillos que busquen el triunfo de la justicia para él, no tiene gobierno que no sea ciego y sordo a sus ayes de agonía, no tiene amigos fuertes a no ser que algún día el público en general, en masa, se levante para desvanecer la sospecha de que en el Perú los corazones son de piedra (Mayer 1916, s.p.).

Se observa, pues, un vaciamiento doble: por un lado, del referente "Gutiérrez Cuevas", pues Mayer no lo reconoció como defensor del campesinado ni mucho menos; por otro lado, de la fantasía "Rumi Maki”, ya que niega cualquier importancia significativa de una restauración del Tawantinsuyu y la m’askaypacha. La Asociación Pro Indígena negó la idea de una sublevación campesina y, más bien, consideró que los sucesos de San José eran, en realidad, manifestaciones de una conspiración gamonal. En mi opinión, esta postura destaca una de las principales características del discurso indigenista, esto es, la imposibilidad de que la población andina posea agencia propia para exigir por ella misma sus derechos. El indigenismo, en todas sus variantes, fue una posición estratégica y de apropiación de una realidad (la "indígena") por parte de un sector ilustrado. Dora Mayer, por esta razón, no podía escapar del tipo escritural indigenista para abogar legítimamente por el campesinado.

Quisiera detenerme por unos instantes en la conclusión de la nota que vengo comentando, ya que allí encuentro una alegoría que me permite pensar en el nominal Rumi Maki Ccori Zoncco. Dora Mayer, en las últimas líneas de "La masacre de San José", escribió acerca de su sospecha de que en el Perú no hubiera más que corazones de piedra. Paradójicamente, como se indicó anteriormente, la traducción castellana del seudónimo Rumi Maki Ccori Zoncco es "Mano de piedra, corazón de oro". Resalto este detalle debido a que en el vocablo quechua se conjugan dos connotaciones que, precisamente, son las abstracciones que Mayer le está negando a Gutiérrez 
Cuevas: piedra y corazón. Considero que estos significantes alegorizan algunos presupuestos que Mayer quiso llevar a cabo en su breve nota. Me estoy refiriendo en particular a las ideas de rectitud ante la justicia y de amor hacia la población andina. Evidentemente, para ella ni lo primero ni lo segundo estuvieron materializados en Gutiérrez Cuevas y mucho menos en Rumi Maki.

Aunque la modalidad escritural de Mayer pueda caracterizarse como indigenista, debo reconocer que entre las diversas personalidades integrantes de la Asociación hubo matices de esa postura. El caso emblemático, para mis propósitos, fue sin duda Francisco Chukiwanka $a^{21}$, quien cumplió un papel trascendental en la visibilización de la problemática campesina de Puno y, además, fue clave para que Dora Mayer — quien radicaba en Lima - pudiera acceder a los sucesos de San José (y a otros, claro está) de primera mano. Para Carlos Arroyo Reyes, Chuquiwanka Ayulo hizo por aquellos años un periodismo de denuncia y utilizó la tecnología de los cablegramas para elaborar notas e informes que envió a Puno, Arequipa y Lima (2005: 180). En efecto, parece que él fue el artífice de la posición que terminó asumiendo la Pro Indígena (negando la sublevación), a partir de la representación de la población campesina como desvalida y siempre víctima. Aunque no es propósito central de mis reflexiones, sí desearía destacar que tanto él como Gutiérrez Cuevas tuvieron una relación de amistad que, a la luz de Augusto Ramos Zambrano (2016), permaneció hasta la muerte del segundo.

Si tuviera que sintetizar el tipo escritural de la Pro Indígena, diría que ella recurrió a fundamentos que fueron coincidentes con los postulados del Gutiérrez Cuevas subprefecto, en otras palabras, a la idea de que la defensa de la población andina tenía que darse en los espacios institucionales, regulados por las leyes y en los que primara el orden estatal. Con este objetivo, al menos en el discurso que defendían, se observa la desconstrucción de la retó-

${ }^{21}$ Francisco Chukiwanka Ayulo fue un destacado indigenista puneño de inicios del siglo XX que, entre otras cosas, se encargó de la elaboración de una utopía lingüística que él denominó “ortografía indoamericana”. Asimismo, tuvo una participación en el Boletín Titikaka (1926-1930). 
rica incaísta que resguardaba Mariátegui. Mayer y Chuquiwanka, estratégicamente, rechazaron el imaginario tawantisuyense y, por ende, negaron la existencia de un líder de inspiración incaica como Rumi Maki. En resumidas cuentas, los tipos escriturales incaístas e indigenistas se distinguieron por las aspiraciones que cada uno perseguía. Naturalmente, los indigenistas pretendieron mejorar la situación del campesinado con una directa intervención en el orden público, a diferencia de los incaístas.

Esa misma nota de Mayer, "La masacre de San José”, presentó una argumentación de antagonismo simultáneo. Es decir, Mayer desdobló su nota en una primera dirección, su matriz, que tenía como norte la postura de la asociación: que en San José había acaecido una masacre (defensa del campesinado). No obstante, de manera simultánea le opuso una segunda dirección: que el gamonalismo era el conspirador de esa masacre (ataque a las oligarquías provincianas). Para el desarrollo de esa argumentación, se valió del periódico gamonalista El Indio, propiedad de Angelino Lizares. Dora Mayer denominó a esa publicación “bastardo periódico”, ya que se estableció como la herramienta discursiva predilecta para defender los intereses gamonales, pero desde un planteamiento singular; en otras palabras, desde una posición que al mismo tiempo que defendía al gamonalismo lo atacaba por enemistades particulares. Solo de esta manera Lizares y Bernardino Arias, gamonales de la misma condición, estuvieron enfrentados. El Indio fue el órgano que visibilizó todas estas contradicciones.

José Tamayo Herrera (1982) estudió con detalle la "mentalidad gamonal” y aseguró que esta tuvo un par de connotados pensadores que elaboraron cierta doctrina gamonalista. De entre ellos, quizás el más destacado haya sido José Sebastián Urquiaga ${ }^{22}$ con su texto Las sublevaciones indígenas en el departamento de Puno que escribió en 1916. Sería interesante detener la mirada en la parte última de ese texto, aunque solo podamos acceder a él por medio de una copia

22 Tamayo (1982) aseguró que, además de Urquiaga, Pedro Irigoyen y Angelino Lizares Quiñones también formaron parte de esa intelligentsia gamonal. 
titulada Indios (Puno-1916). En tal sección, llamada "Sublevaciones de indios”, se reforzó la idea de la gran sublevación de Gutiérrez Cuevas, el cual es equiparado a Rumi Maki. El detalle de la sublevación de San José que Urquiaga brindó es sumamente referencial, pues incluso recurrió a datos cuantitativos (número de armas que supuestamente habría empleado el "ejército incaico") para reforzar un tipo escritural gamonalista.

Además de la información anterior, Urquiaga trazó una “secuencia” temporal de las sublevaciones campesinas que inició — según él mismo- con el levantamiento de Juan Bustamante (1866). Esta inserción histórica, luego, fue el punto de apoyo de la retórica gamonalista que Urquiaga empleó. Es decir, utilizó la figura de Bustamante como equivalente semántico de "restaurador incásico", con el fin de situar nominalmente la idea "tawantisuyense”. De aquí, trazó otra equivalencia, aunque esta vez de índole histórica: Teodomiro Gutiérrez Cuevas (Rumi Maki para el propio Urquiaga) era la actualización de Bustamante. Al haber asentado la afirmación de que el levantamiento de San José era un hecho de problemática social, él mismo reconocía que en Puno existía un enfrentamiento clasista. A pesar de las riñas intestinas dentro del propio sector terrateniente (por ejemplo, Lizares y Arias), lo que pretendía el tipo escritural gamonalista era caracterizar la presencia de Rumi Maki como perturbadora y portadora de odio.

Dicho a modo de síntesis, el texto de Urquiaga, amparado en el seudocientificismo, deseó realizar un balance crítico de la situación del campesinado en relación a la clase terrateniente. Entonces, bajo la lupa gamonal sí hubo un restaurador mesiánico de nombre Rumi Maki, quien montó no solo un discurso de subversión tawantisuyense, sino también un plan de acción fáctica: “Gutiérrez para llevar a efecto su plan invistió del rango de general, cambió su apellido por el nombre quechua Rumi-Maqui [...] repartió nombramientos escritos a los cabecillas o jefes de movimiento. Estos nombramientos llevan el sello del nuevo Estado Federal del Tahuantinsuyo" (Urquiaga 1977: 50). 
Todo este plan —el cual desarrolló con minuciosidad Urquiagatiene varios pliegues complementarios que le otorgaron a la retórica gamonalista especial importancia dentro de los tipos escriturales que se dedicaron a Rumi Maki. Uno de esos pliegues es el que se refiere a la victimización de los hacendados, cuya lectura se sigue a partir de los asaltos campesinos que Urquiaga relató: "Y en tal virtud el $1^{\circ}$ de diciembre inició el ataque con los indios que pudo reunir [Rumi Maki], sobre Atarani, hacienda del señor Alejandro Choquehuanca, vecino de Azángaro la que fue talada y destrozada" (Urquiaga 1997: 51). El ejercicio escritural llevado a cabo por el autor de Indios (Puno - 1916) posee, además, otro pliegue semántico interesante. Me refiero a la aparente objetividad con que se pretendió leer el fenómeno de la sublevación: el texto de Urquiaga llama la atención por la abundancia de datos y por los sistemáticos paralelismos allí presentes: Juan Bustamente/Rumi Maki, Tawantinsuyu/utopía de ignorantes, pobladores andinos/idólatras, etc.

Esos dos pliegues le permitieron a Urquiaga materializar un tipo escritural que guardó profundas y evidentes diferencias con las representaciones incaístas de Mariátegui pero que, además, entró en colisión con la retórica indigenista alentada por Chuquiwanka y Mayer. Si bien estas últimas reconocieron la existencia de una sublevación, su interpretación de los hechos fue marcadamente contrapuesta. Por esta razón, la figura de Rumi Maki (peligro real para el gamonalismo y artificio perverso para el indigenismo) sirvió de parteaguas y de modelador escritural. Para Urquiaga y su sector, Gutiérrez Cuevas fue el mismo Rumi Maki y pretendió alzarse para la instauración de un estado del Tawantinsuyu. En cambio, para Mayer, Chuquiwanka y los proindígenas todo lo anterior fue una invención del gamonalismo con el propósito de consolidar su dominio en el sur peruano.

Como puede verse, a diferencia de la Pro Indígena, el discurso gamonalista incentivó la idea de la restauración tawantisuyense con el fin de afirmar, a la vez, su propia posición de dominación. La realidad sobre la que situó su texto Urquiaga puede ser entendida bajo la lógica de una lucha social, es decir, bajo un modelo en el que el 
gamonalismo llevara a cabo la dirección de las tierras. De este modo, el tipo discursivo que sus representantes utilizaron estuvo amparado en la victimización del terrateniente y, en consecuencia, en la demonización del campesinado. Aunque en este punto los "proindígenas" y los gamonalistas tuvieron posturas contrapuestas, lo cierto es que, para ambos sectores, la creencia de la mask'aypacha simbólica era una locura propia de las mentes de un Tawantinsuyu arcaico.

\section{Palabras finales}

En este trabajo, como ya he comentado en la presentación del mismo, he pretendido incidir en las implicancias de los tres tipos escriturales que representaron a Rumi Maki. De esta manera, procurando evitar la verificación histórica, he querido subrayar las características más notorias de cada registro (incaísta, indigenista y gamonalista) con el propósito de contrastarlos y de asemejarlos. En última instancia, he querido demostrar las semejanzas que se tuvieron los intelectuales, escritores y gamonales que optaron por volcarse a la defensa de las poblaciones andinas. Sin embargo, también he intentado situar a cada uno de ellos dentro de una lucha particular y de un interés también propio. Por último, desearía mencionar un aporte no menor de mis reflexiones. Me refiero a que, por primera vez, se presenta una lectura detenida de las retóricas o modalidades de representación que suscitó la existencia de un restaurador inca de nombre Rumi Maki.

\section{REFERENCIAS BIBLIOGRÁFICAS}

$\mathrm{S} / \mathrm{N}$

"Reportaje al mayor Teodomiro Gutiérrez Cuevas". El Pueblo. Arequipa, 17 de enero de 1917.

Arroyo Reyes, Carlos

2005 Nuestros años diez: la Asociación Pro-Indígena, el levantamiento de Rumi Maqui y el incaísmo modernista. Buenos Aires: LibrosEnRed. 
BUSTAMANTE, Luis

1987 Mito y realidad: Teodomiro Gutiérrez Cuevas o Rumi Maqui en el marco de la sublevación campesina de Azángaro (19151916). Memoria de Bachiller en Historia. Lima: Pontificia Universidad Católica del Perú.

Chuquinuanca Ayulo, Francisco

1916 "Relación de los hechos realizados en Azángaro el 1 de diciembre de 1915”. El Deber Pro-Indígena. 40, 203-208.

Flores Galindo, Alberto

1987 Buscando un inca: Identidad y utopia en los Andes. Lima: Instituto de Apoyo Agrario.

GonzÁlez Prada, Manuel

[1905] 1986 “Autoridad humana”. En Prosa menuda, tomo II, volumen 4. Lima: Copé, pp. 299-230.

Mariátegui, José Carlos

1917 “Grimas y zozobras”. El Tiempo. Lima, 17 de enero.

Mariátegui, José Carlos

1917 “Minuto solemne”. El Tiempo. Lima, 25 de abril.

MAYer, Dora

1916 "La trama de la sublevación indígena”. El Comercio. Lima, 3 de enero.

MAYER, Dora

1916 “La masacre de San José (Azángaro)”. El Comercio. Lima, 14 de enero.

Paredes, Mauro

1970 "El levantamiento campesino de Rumi Maqui (Azángaro, 1915)”. Campesino. 3, s.p.

Ramos Zambrano, Augusto

2016 “ Rumi Maqui”. En Ezequiel Urviola y el indigenismo puneño: Tormenta altiplánica, Rumi Maqui y la Rebelión de Huancané. Lima: Fondo Editorial del Congreso.

Rodríguez Toledo, Luis

2015 "Rumi Maqui en la historiografía peruana, desde Mariátegui hasta el siglo XXI". En Antología del indigenismo puneño. Puno: Universidad Nacional del Altiplano. 
TAmayo Herrera, José

1982 Historia social del indigenismo en el Altiplano. Lima: Ediciones Treintatrés.

URQUiAgA, José

1977 Indios (Puno-1916). Lima: Universidad Nacional Mayor de San Marcos.

VAlDELOMAR, Abraham.

1917 “¡Por la Gloria de la raza!”. La Prensa. Lima, 26 de febrero.

VAlDELOMAR, Abraham

1917 “El triunfo de la raza!”. La Prensa. Lima, 28 de febrero.

Velasco Aragón, Luis

1986 "Comentarios inéditos sobre Teodomiro Gutiérrez Cuevas". En Prosa Menuda, tomo II, volumen 4. 